\title{
Overcoming Dyadic Boundaries: Reading Poetic Experience after the Semiotics and Pragmatism of Charles S. Peirce
}

Tiago da Costa e Silva $a^{1,2, *}$

${ }^{1}$ Cluster of Excellence Image Knowledge Gestaltung: An Interdisciplinary Laboratory, Sophienstraße 22a, 10178 Berlin, Germany

${ }^{2}$ Department of Cultural History and Theory, Institut für Kulturwissenschaft, Humboldt Universität zu Berlin, Georgenstr. 47, 10117 Berlin, Germany

Received April 17, 2018; Accepted August 23, 2018

\begin{abstract}
The aim of this article is to offer a reading of the poetic experience through the scope of the semiotics and pragmatism of Chares S. Peirce. Such a reading through semiotics and pragmatism unveils deeper levels of the process of interpretation involving abduction, an inference through which new meanings implied in the semantic tensions arise. Methodologically, the article begins with Roman Jakobson's realisation that only a broader semiotical context, which breaches the boundaries of the dyadic components of significant and signified scope of structuralism, enables the access to deeper levels of poetic events. The article's author then discusses the limitations of the dyadic relations of structuralism and, as a broader processual framework to assess poeticity, sets out to discuss the poetic experience from the perspective of pragmatism and its all-encompassing logic of abduction...
\end{abstract}

Keywords: semiotics • methodeutic • poetic function • dyadic structure • poeticity • logic of abduction • pragmatic reading

(C) Sciendo

\section{Introduction: Insight of Poetics and the Need for an Expansion}

The present article's main objective is to offer a reading for the poetic experience through the scope of the third branch of the semiotics of Charles S. Peirce. In this third branch, which Peirce called methodeutic, the philosopher fully articulated his maxim of pragmatism by the symbiotic functioning of the inferences of abduction, deduction and induction in a methodological manner. As a maxim of logic, pragmatism is, according to Peirce, contemplated as an open-ended theory of inquiry, which is driven by the logic of abduction, because every newly discovered element that enters a semiotical process, and thereby possesses the character of a hypothesis, should be capable of entering the process of experimentation as well. The present article's main thesis runs as follows: abduction, as a living process of discovery, introduces a newly given element into semiosis through the hypostatisation of relations, that is to say, by the process of rendering a highly suggestive and yet vague new idea more clearly and distinctly to the perceiving mind, thus allowing conceptions and conceptualisations to be formed. It is therefore important to note that this aspect of abduction, which is connected to pragmatism's capability of integration and differentiation, is a characteristic feature observable in any poetic experience or manifestation of poeticity.

In this paper, I depart from Roman Jakobson's insights for the need for a disciplinary expansion and also a paradigmatic change, thus departing from the semiotical and semiological systems drawn from the more general scaffold of structuralism. This exact point of departure consists of Jakobson's assumption of a realm of a general science of signs encompassing all varieties of languages and sign systems with 'pansemiotic features'. Jakobson's insight for the need of this disciplinary expansion and paradigmatic change can be seen as an indication of a fruitful perception that led him to search for a much broader openness towards the consideration of poeticity. This expansion and change in paradigm, I believe, can be achieved with Peirce's theory of inquiry, with especial regard to the phaneroscopy (or phenomenology), semiotics and pragmatism in an integrative and systemic way.

Now, it is important to note that Jakobson had some knowledge of Peirce's theory of signs, i.e., the speculative grammar, at some extent, although Jakobson reinterpreted and reframed some of these concepts within his more structural theoretical framework [1 p. 45]. My intention here is not to make a parallel of the interpretation and compatibility of theories between Jakobson and Peirce but to expand upon Jakobson's insight for the need of the aforementioned paradigmatic expansion set off by the studies on poetic experience. I intend to skip, thus,

\footnotetext{
*Corresponding author: Tiago da Costa e Silva, E-mail: tkunst@gmail.com 
the theoretical difficulties of Jakobson's reinterpretations of Saussure and Peirce's classifications of signs, i.e., speculative grammar; I focus on the critical analysis of the general aspects of structuralist-based sign theories and reclaim this change of paradigm with the theoretical framework of Peirce's methodeutics and pragmatism, that is, with Peirce's more processual branch of his semiotics.

From this perspective, some concepts of Jakobson can be systemically compatible with that of Peirce, though not directly, but processually. Here, I am referring to the studies in iconicity and poeticity that can accommodate analysis from both Peirce's theory of signs and Jakobson's specific theory of poetic language function. By no means, I am here declaring that Peirce's concept of sign and semiosis is directly translatable and directly compatible with structural and semantic sign systems. In this paper, I am pointing out, however, that some investigations of poetic experience, such as Jakobson's inquiries into poetic messages, have the potential not only to disclose at some extent the need for a more plastic semiotic approach but also presupposes and in a way requires such an approach, so as to grasp in a fuller way the significative potentials of such poetic experience.

It is fairly known that the main scaffold of structuralism consisted of a rather heterogeneous field of knowledge production and consequently the various studies of language systems, although based upon basic premisses of the duality of the conception of sign as a composite entity, differed significantly in their consideration of this entity. There have been modifications of and derivations from initial assumptions of Ferdinand de Saussure and from his early attempts to formulate, at least at some extent, a project for his newly conceived, but not finished, discipline of semiology [2 p. 168]. There are, consequently, distinctive changes in framing the unity of the sign and its function within certain specific linguistic, formalistic, or structuralist framework. For example, Saussure's semiological model is based upon a dualistic notion of the composite entity of the sign or, as he affirms, upon a 'dual psychological entity' consisting of the sign that Saussure calls signifier and an 'idea', which he calls the signified, that is, the meaning of the sign [2]. ${ }^{1}$ As Saussure affirms:

We can therefore conceive of a science which studies the life of the signs within the social life; it would form a part of social psychology, and consequently of general psychology; we will name it semiology (from Greek sēmeĩon, 'sign') ([2 p. 84]. My translation). ${ }^{2}$

Jakobson has a different approach to this unity, in which, for him, this dual entity is made of a signans and a signatum. Even though there are crucial differences between Jakobson's and Saussure's conception of sign, as there is also such differences among other lines of inquiry into sign theories, such as Hjelmslev's, Greimas', Lotman's, Eco's, Barthes', just to quote a few, these conceptions share the characteristic of being based upon a dyadic form of functioning. ${ }^{3}$ Whereas it is not the task of the present paper to dwell upon the inherent and particular differences concerning these sign systems and theories, there is one aspect that, apart from the specific differences of such systems, hallmarks the general structuralist purview: the dyadic aspect related to the composite structure of the conception of sign and the consequent dyadic boundaries of the functioning of such structural scaffolds.

I intend, therefore, to critically analyse, in very general terms, the dyadic boundaries of sign theories based totally or at least to some extent - on structuralism. I will not, however, as aforementioned, study the specificities inherent to the distinct theoretical models within structuralism, since this field is by no means homogeneous. Therefore, I proceed to thematize Jakobson's insight of expanding the framework of sign theories provoked by poetic experience, thus enabling a better understanding of what Jakobson meant as 'pansemiotic features', with the much broader theoretical framework of the semiotics and pragmatism of Charles S. Peirce.

1 As I have shown elsewhere, there has been a common tendency to mixture the distinct sign systems, and this enabled the ensuing chaos regarding a pastiche of many forms of sign systems and sign theories in general, forms of semiotics and semiologies [3]. A thorough analysis of the main characteristics of the theoretical specificities of sign systems and the differences between these theories goes, however, beyond the scope of this paper.

2 From the original in French: 'On peut donc concevoir une science qui étudie la vie des signes au sein de la vie sociale; elle formerait une partie de la psychologie sociale, et par conséquent de la psychologie générale; nous la nommerons sémiologie (du grec sēmeĩon, «signe»)' [2 p. 84].

3 As to the fundamental differences between dyadic-based systems and Peirce's theory of semiotics, one could remark that the distinction lies on the dyadicity of structural systems on the one hand and on the tradicity of Peirce system on the other hand. This distinction, alone, is not, however, enough to correctly understand the distinct functions of both positions. As Thomas Short mentions: 'it is a basic error to suppose that the difference between the two concepts of sign is simply that one is dyadic and the other triadic. Saussure made the sign a dyad, a two-sided entity. Peirce, on the contrary, made the sign just one relatum of a triadic relation, of which the two other relate are the sign's object and the sign's interpretant. All three items are triadic in the sense that none is what it is - a sign, an object, or an interpretant - except by virtue of its relation to the other two' [4 pp. 18-19]. 
The word overcoming is being here used to reinforce this aspect of the semantic tension included in the insight of 'pansemiotic features'. It is the internal tension of a poetic experience itself that provokes the tension along the borders of a given sign theory. Consequently, a more powerful theoretical scope will necessarily be required to grasp the generation of poetical meaning produced by this tension, given that poeticity has, in itself, the power to overcome previously established and thus sedimented semantic structures. The point I am making in the paper is not that there must be a shift from dyadic semantic structures to a processual triadic sign function. The point here is that the poetic experience will force the structural barriers, will overcome it, and a broader plasticity will eventually come into play. This will demand for a much broader semiotic approach. Peirce's theory of inquiry can meet this demand, in which it offers here a broader systemic scope for the study of poeticity, for it, poeticity; will enter the whole semiotical process as experience, that is to say, as phanera ${ }^{4}$ and then will enter the realm of semiosis. As such and because of the given processuality that poetic experiences bring with themselves, there will be a strong movement of new interpretative articulations at work in the third branch of semiotics, that is, methodeutics, which is the branch of semiotics in which the maxim of pragmatism, guided by the logic of abduction, becomes active. This point will be discussed in depth in the third section of this article.

Here, I must stress that I do not mean to disregard other sign systems by claiming that they are completely illsuited to articulation within the context of poetics or generative processes or by maintaining that Peirce's semiotics is the only theory that can satisfactorily explain poetic experience and poeticity from this angle. I do argue, however, that, for the task at hand, Peirce's semiotics offers both a set of general theoretical principles and a characteristic processual openness that enable the inquirer to consider the poetic experience in the most optimal manner in order to offer an explanation for the transgressiveness of such poetic experience, which, in its turn, enables anything with the character of poeticity to constantly breach closed systems and overcome boundaries.

\section{I - An Insight for the Need for Expansion in the Field: The Transgressive Feature of the Poetic Experience and an All-Encompassing Perception of Semiotics}

Roman Osipovich Jakobson was one of the most important and influential linguists and semioticists of the twentieth century. Though he humbly referred to himself as a 'philologist of Russian language', Jakobson was a polymath who contributed, for instance, to the research of language, semiotics, cybernetics and semiology. At the beginning of the twentieth century, he was one of the founders of Moscow's linguistic circle and an influential participant of the poetry group Opoyaz, an acronym formed from the initial letters of the Russian words for 'society for the study of poetic language', or Общество изучения Поэтического Языка, ОПоЯз. During his early career at the beginning of the twentieth century, he focussed on the research of language from a purely formalistic point of view. At this point in time, Russian formalism was not yet a semiotic current; it dealt with questions of aesthetics and the theory of literature. Jakobson later became a member of the linguistic circle of Prague and, in making use of structuralist principles, contributed significantly to the field of textual semiotics and aesthetics. Although the name of this school of thought implied the predominance of linguistics, Prague's linguistic circle also embraced phonology, the function of speech sounds as well as textual linguistics [5 p. 100]. The results achieved, however, led the group to supersede disciplinary barriers and to include other theories, especially those originating in other sign systems. For instance, the discovery of distinctive characteristics of the phoneme as a sort of 'minimal particle' or 'atom' of language, combined with the principles of phonological analysis, helped to create models of analysis able to function and to be articulated with other models of sign systems [5]. Within this prolific environment, Jakobson contributed to the research in the fields of the semiotics of aesthetics, literature, poetics and poetical processes as well as the semiotics of style and stylistics. Based upon the theoretical scaffoldings of structuralism, especially upon the basic principles of Saussure and Hjelmslev, the linguistic circle of Prague aimed at the research of language processes within the act of communication, that is, the research of language and its elements within the dynamic context of communication. In other words, the objective was to study language as it functions and in its production of meaning, both synchronically - that is, the functioning of a language as it is - and diachronically - that is, the functioning of a language as it changes. In this new approach, language was seen and studied as a dynamic, functional

4 Peirce uses the term phenomenon, derived from the Ancient Greek paivó philosophical developments. Phaneron is also derived from the Ancient Greek word pavepós, (phanerós), which means 'manifestation', 'visible' or 'perceivable through the senses'. Peirce uses this word to mean that element that appears in an interpretative mind in any form. 
system. Jakobson recognised that it is impossible to consider communication without taking into account other interconnected realms, such as the social environment [6 p. 43, 5 p. 103]. He says:

[...] the question of relations between the word and the world concerns not only verbal art but actually all kinds of discourse. Linguistics is likely to explore all possible problems of relation between discourse and the 'universe of discourse': what of this universe is verbalized by a given discourse and how it is verbalized - to say with the logicians - 'extralinguistic entities', obviously exceed the bounds of poetics and linguistics in general [7 p. 351].

Roman Jakobson defines language in its broadest sense to include verbal and non-verbal languages, as well as all kinds of sign manifestations, which can potentially bear, convey and translate some significance. In his later work, he identifies the need to expand the research field regarding the articulation of languages, especially in what refers to poetic manifestations. As an avid researcher, he was able to explain poetic polysemy from a semiotic standpoint, one that would involve a specific type of semantic and pragmatic tension within a certain language system as well as between the language system and diverse reference points external to - but somehow connected with - the language system in question. In a quote from his famous text 'Linguistics and Semiotics' (1960), Jakobson states that:

[...] poetic features belong not only to the science of language but to the whole theory of signs, that is, to general semiotics. This statement, however, is valid not only for verbal art but also for all varieties of language since language shares many properties with some other systems of signs or even with all of them (pansemiotic features) [7 p. 351].

Instead of being encapsulated in a narrow definition as referring only to well-codified systems, the term language shall be here considered in its broadest sense, meaning not only the codified verbal languages but also general forms of non-verbal languages with different degrees of codification. Being in the world, surrounded by an intricate net of languages, forces the interpreting minds to communicate with it through the reading (decodifying) as well writing or graphing - that is, writing with some technique in some medium (codifying) - of forms, which are, in their vast majority, codifications and proto-codifications of non-verbal languages, such as noises, images, visual signs, acoustic signs, odours, gestures, facial and corporal expressions, social movements, clothes, costumes, cultural aspects and natural signs. The term synesthesia can refer to the vast set of non-verbal signs that could, through successive acts of associations, rearrangements and re-signification in distinct contexts, possibly spark more codified languages. Through the interactive process of translating, interpreting, rearranging and re-signifying, the languages can grow. After experiencing these aspects of non-verbal languages and realising that they in fact play a major role in the processes of both cultural formation and humanisation, Ernst Cassirer affirmed in his book An Essay on Man [8] that a human's essence and humanity do not consist of hard, deductive ratiocination - which the eighteenth-century Enlightenment philosophers defended as the most important asset that differentiates humans from other species - but the capacity of communicating other forms of signs and thereby sharing with each other levels of signs, thus acquiring more sensibility through these exchanges.

Jakobson perceived the need to overcome the hard dualism at the core of structural linguistics and semiology. He devised theoretical models as a means to solve this difficulty, which led him to include other systemic relations that are revealed by the communicational act. He devised a general model that represents the dynamics of communication by indicating the essential elements articulated in such an act, as well as the subsequent language functions that are derived from it. This dialogical model, though focussed on the dynamics of speech, can be extended and articulated in non-verbal communicational models as well. According to Jakobson, the model can be described as follows (Fig. 1):

The addresser sends a message to an addressee. In order for this to work, the message needs to have a context or referent. The context must be comprehensible and structured in a certain form so as to be properly understood. Furthermore, there must be a code, which has to be at least partially or totally common to both the addresser and the addressee in order for the message to be encoded and/or decoded. The process of communication is made possible by the category of contact, which is, according to Jakobson, a physical channel and a psychological connection between the addresser and the addressee through which the communication can be supported [5 p. 105, 7 p. 353]. 


\section{CONTEXT}

ADDRESSER MESSAGE ADDRESSEE

CONTACT

CODE

Fig. 1. Roman Jakobson's diagram representing the main elements present in the communicational process.

From these six elements that comprise the dynamics of the communication act - that is, the addresser, the addressee, the context, the message, the contact and the code - six functions of language emerge, each of which refers to the predominance of one of the aforementioned six elements. The particular emphasis defines what kind of language function is being articulated. An act of communication can exhibit more than one language function, but only one of them is predominant at each act of the communication process. This is referred to as the principle of dominance. The predominant language function forces the others into the background. All six functions, however, are essential to the process.

According to this dynamic of the act of communication, if the process is adjusted to focus on the referent, or context, the referential function will be predominant. The referent or context is that which is indicated by the message. Nöth [5 p. 105] affirms, for instance, that this language function predominates in descriptive texts as well as in reports or descriptions.

The expressive (or emotive) function is predominant whenever the message is directed towards the addresser but not necessarily focussed on the message's contexts. Examples of this are forms of expression in which exclamations or euphoric forms are common [5].

The appellative or conative function is predominant in the process of communication whenever the message is directed towards the addressee. Vocative and imperative expressions, as well as gesticulations with a pointed finger towards someone, are examples of this. The conative function is also the main form of communication used in advertising, for instance.

The next language function is the phatic. Jakobson took the concept of phatic from the Polish anthropologist Bronisław Kasper Malinowski, who considered this specific act of communication as a simple exchange of words or even sounds, by means of which social relations can be produced, without the need for a determinate content [5 $p$. 105]. Jakobson appropriated this concept and used it to describe acts of communication that function to establish a contact, to prolong an act of communication or to interrupt a given act. Examples of this are communication processes in which greetings and polite forms of addressing someone are uttered in order to establish an act of communication. Sentences of the types 'Hello? Do you hear me?' are also types of phatic functions of language, for they act to establish, re-establish or assure the continuity of an act of communication [7 p. 335].

The metalinguistic function is predominant whenever the focus of the act of communication is the language and act of communication itself. According to Nöth [5 p. 106], Jakobson determined that logical semantics possesses two levels. The first level is the objective language, which is language articulation directed towards extra-linguistic references, that is, to use the language to discuss something else. The other level of logical semantics is that of meta-language, which is language articulation that refers to language itself. For instance, all forms of discourse about grammar, lexicography, etymology, terminology, orthographic rules and definitions are always related to the level of metalanguage. It is possible to encounter such functions in most situations of everyday life. Whenever one expresses 'What do you mean?' or 'l'm not following you', the predominance of the function within the act of communication is metalinguistic.

The sixth language function is the poetic function of language. Whenever the act of communication is directed to the message itself, poeticity is predominant. According to Jakobson, the poetic function, or poeticity of an act of communication, appears whenever some special combination comes into being. When an element, say a noun, is selected and inserted into a different context, a certain semantic tension is created between these two substantives that cannot act separately but only within the new context and only by being structured in a sequence. This adds tension to the semantic dimension to imply other significations than the one first implied, thus expanding the level of paradigms, the meaning of the two nouns, into this new combination. There is a tension between the separated strata 
of a paradigmatic act of communication and the newly formed combination. This requires a semantic extension of the new combination. The semantic articulation triggers operations of similarity, which thereby create a kind of 'ludic game' and allow the new combination to acquire multiple and multilayered possible significations.

In the perspective of structuralism, the manifestation of something 'poetic' within a system of language is explained by the projection of the principle of equivalence from the axis of selection into the axis of combination. Moreover, a second combinatorial operation takes place, that is, the projection of the principle of contrast from the significative, selective and combinatorial operations into the level of the 'palpable' and 'perceptible' forms [9 pp. 129-132, 10 p. 65].

Taking for instance the phrase Juliet is the sun, the interlocutor is confronted with a myriad of possible interpretations because the message becomes centred in itself, that is, it refers to its own content, both in its structural elements and in its global setting as a message. Thus, it is clear that a semantic tension is created, for there is no definitorial rationale for understanding this sentence other than to face the tension itself. Juliet is a feminine name, whereas the sun is another substantive used here as a predicate of the name. So, the semantic operation proposed by the sentence forces the reader or interlocutor to interact with this tension and allow it to be the producer of associative meanings. No rational explanation will enable a complete disclosure of the poetical meaning intrinsic to this sentence. Thus Juliet is the sun provokes multilayered and polysemic effects that occur through the semantic tension by forcing new combinations of selected paradigmatic structures [5 p. 450, 11 pp. 68-72]. This tension provokes new semiotic operations - not only semantic ones - because the forced similarities created by the initial tension pervade all forms of operations of similarities in processes of thought and in perceptive comparisons. This can be explained by making use of diverse theories of poetic or aesthetic metaphor [12]. However, as I have indicated in the "Introduction: Insight of Poetics and the Need for an Expansion section", I chose to work with the third branch of semiotics instead of being concentrated mostly on the classification of signs. Given the fact that metaphors, in Peirce's semiotics, are specific types of icons presenting relations of structural analogical relations with some object, the relation to pragmatism would be rather laborious to accomplish within the scope of a single article. By framing the present inquiry through the scope of methodeutic and pragmatism, I intend to show that the entirety of semiotic processes, linguistic and non-linguistic alike, will be triggered by the poetic experience and will, therefore, become affected by the poetic expression at the pragmatic level. As I will show, poeticity eludes the structuralist encapsulation and frees the senses towards further semiotic processes. Perhaps, these discoveries have led Jakobson to consider poeticity as a factor in the act of communication that is more open to the realm of semiotics - here considered as a general theory of all types of signs and sign processes.

As Umberto Eco states in his article 'Roman Jakobson and the Development of Semiotics' [6 p. 122], 'in every sign exchange there are not only isolated items: semiotics must, as does contemporary linguistics, shift from a theory of single terms and "phrases" to a co-text and context theory. This also means that semiotics should incorporate not only syntactics and semantics but also pragmatics'. In this case, I believe, Roman Jakobson aimed at proposing this paradigmatic expansion, when he affirmed, as aforementioned, that poetic features extrapolate the science of language and can be studied only from the broader perspective of a general theory of signs apt of grasping the verbal and the non-verbal, for the poetic features share a myriad of properties with many other systems of signs - or may be with all of them [7 p. 351].

However, a problem with the structuralist purviews remains. The poetic experience, when viewed and explained from structuralist positions, fails to grasp deeper levels of poeticity, especially those related to extra-linguistic levels and levels of formation and association of information that precede the inclusion of such information into a determined structured system of a sign and a thing signified. There is a prevalent dualism within structuralist systems that seeks to encapsulate poetic experiences by only describing the semantic tensions created in the system. All else - such as formative processes, extra-linguistic experience, phenomenological experiences and aesthetic experiences connected with the poeticity in question - is left aside, because dualistic structures are not made to hark that far outside the hardened structural-linguistic-based systems. In the next section, I refer in detail to the problem of dualism within structurally based sign systems.

\section{II - Dyadic Boundaries: Questioning the Rigid Frontiers of Structuralism}

As I am proposing in this paper, the inquiry into the poetic experience with the theoretical framework of the semiotics of Charles S. Peirce demands a new approach, one that differs from the common one of the structural and linguistic-based sign theories. Here, I intend to justify this claim. The traditional approaches utilising sign theories 
and semiologies based on structural linguistics and structuralism are not up to the task at hand for two main reasons. In the first place, they are mostly limited to the scope of codified - i.e., already conventionally formed languages. Their applied methodology requires the atomisation and subdivision of a conventional language system into verifiable structures and subcodes. Methodologically, this becomes 'a technical means to designate different objects in a homogeneous way', because the verifiable 'structures' are models 'constituted according to operators of simplification which allow the unification of different phenomena from a single point of view' [13 p. 63]. This results in the theories of this purview tending to explain everything as codified and conventional language functions. In central aspects, Peirce's semiotics contradicts these forms of dualistic, structuralist sign theories, for its theoretical scope and explanatory powers are not limited to the realm of conventional significations and linguistic structures. More specifically, it is possible to say that Peirce's theory of inquiry has a very different nature, especially in regard to the conception of sign, as aforementioned. Peirce's concept of sign is inasmuch different from that proposed by structuralist purviews because it is not only different in number of items combined - i.e., triad instead of a dyad - but also the way these elements are combined and articulated. It is, thus, the difference between a composite, dualistic entity and a relational, future oriented articulation [4 pp. 18-19]. Peirce's conception of sign involves, furthermore, his phenomenological categories, of which thirdness plays an important role in the characterisation of the sign as a correlatum in a given contextual relation of mediation. This surpasses, for instance, Saussure's conception that considers the composite-like sign to be a psychological product of a purely mental production from an idealisation of language articulation out of a trigger-like signalising component. ${ }^{5}$

If a given object of study escapes the high degree of typically structuralist formalisation and the character of already defined languages as sign systems, as is the case with poetic phenomena or poetic events in general, a mainly structuralist approach becomes not only difficult but rather extremely brittle. To account for such dynamic, formative systems, the structuralist approach must rely on building analogies with a theory, so as to use the theory in a certain way that will enable a specific experience or phenomenon to be grasped by the theory. The problem here is not so much to consider a given work of art - be it an oil painting, a film or the Guggenheim Museum Bilbao as evoking 'multilayered and polysemic' meanings. The problem is to attribute such characteristic features of textual language systems, e.g., multilayered, polysemic textual structure, to other non-verbal and non-linguistic areas based on theories constructed upon analogies. Analogies, approximations and metaphors devised to understand different fields as a form of 'text' in order to fit the structuralist methods came into use to analyse, decompose and explain phenomena. It is typical of structuralist methods, for example, to explain a complex work of art by imposing upon it textual-structural categories and forcing decompositions of the 'textual' forms, such as subdividing it into syntactical, semantic and lexical 'unities', and types of 'discourses'. Such analogical approximations were widely disseminated from the late 1950s through the 1970s. Referring to exactly this point, Johannes Ehrat, in his account of narration and representation in cinema from a Peircean perspective, affirms:

Theory suffers when we adopt methods used for other objects on a metaphorical or analogical basis. This is because we constantly risk overstretching the principle of analogy, the metaphorical point of comparison between two objects. Interpretations based on those analogies in fact jump to conclusions - sometimes even a leap - and they cannot but falter once their metaphorical basis is shaken [14 p. 4].

In the second place, because structuralist sign theories are based upon a dichotomic notion of sign that separates everything into two parts, that is, the signified and the signifier - which is actually derived from structural linguistic methodology [ 5 p. 46] - everything that is considered to be a sign must reflect these two elements. The problem is that these two elements are made into two absolute ideal values, the signifier as signalised part, a mark, and the

5 According to Thomas $\mathrm{L}$. Short, there is still a noteworthy fundamental difference related to the distinct ways in which the sign theories based upon structural purviews and Peirce's philosophical semiotics. He affirms: 'Peirce admitted a variety of grounds of significance, including resemblance and causality. Thus his semeiotic embraces natural signs and images, as well as arbitrary signs. Furthermore, he made particular signs as well as types of signs a primary study. And finally, he admitted that, in addition to thoughts, other responses to signs, such as feelings and actions, can be interpretants. Hence, sign interpreters are not necessarily humans only. This breadth appears to be one of its features that has caused semeiotic to be favored over semiology. However, it does not follow that Peirce's semeiotic embraces Saussure's semiology as a part. If Saussure was right that systems of arbitrary signification can be studied in abstraction from their particular uses and in abstraction from natural signs and other nonarbitrary forms of significance, then Peirce was wrong. And if Peirce was right that language can be understood only in the concrete context of its uses, in cooperation with other kinds of signs, then Saussure was wrong. One system cannot be a part of the other, because one contradicts the other.' [4 pp. 19-20]. 
signified, that is, the idea that is evoked. ${ }^{6}$ Everything else that does not fit into this framework will be disregarded as being unable to function as a sign for something. This is precisely what renders dichotomic semiological theories inapt for the task at hand. For example, many semiologies would not consider specific phenomena - such as unique bluish-metallic marks on a piece of pure of titanium or the particular quality of brush strokes in a work of Monet, the sound of an acoustic contrabass or a spontaneous social movement in the urban environment of 1960s New York City - as potential signs in themselves. As one of the representatives of structuralist semiology, Yuri Lotman states that the same object 'may be studied from a semiotical and from a non-semiotical point of view' [15 pp. 4-5]. This statement reveals the clear duality within structural semiologies and sign theories and their tendency to be reductionist in the sense that a sign system is for the most part related to an already highly codified referential system. Thus, these theories grasp only very specific elements as relevant signs and disregard the rest as being non-signs. Semiologies and sign theories based on structuralism and linguistics fail to grasp the phenomenologically qualitative and indicative aspects of reality in its ubiquitous multiplicity that impose themselves upon the senses [14 p. 6]. Exactly, these latter qualitative aspects of phenomena - such as the qualities present in a piece of titanium or brush strokes on canvas, the dynamic characteristics of a social movement or the characteristic sound of an acoustic contrabass - may be presented as qualities of some unique sign and possible qualities of phenomenological forms that can be semiotically organised into a potential system of language. These 'hidden' qualitative aspects can play a fundamental role in a developmental, formative process, for they are qualities that may turn into repetitions of qualities that, in their turn, start to form a new regularity on the verge of becoming something else - a sort of form-giving process not yet included in any system of language -, as I will demonstrate as follows: an artist experiments with forms produced by oil paint on canvas. He seeks to develop a new visual construction, a new formal pattern in order to create a new visual identity. Upon testing many techniques, he stumbles upon a certain characteristic of paint being distributed on canvas by means of hitting the surface with the brush differently. He realises that the combination of this movement, the quality of paint and the quantity of solvent and oil creates a certain visual effect. He finds it interesting and sets out to improve upon it. In doing so, that which seemed irrelevant from one perspective, indeed, insignificant, becomes the centre of new semiosis or semiotic processes - in Peircean terms.

\section{III - Poetic Experience and the Logic of Abduction: The Poetical Operation Revisited from a Pragmaticist Perspective}

Let me turn my attention to Peirce's methodeutic and pragmatism. Pragmatism, as I here present, is pervaded by the logic of abduction. Now, abduction, as an inference, initiates the process of hypostatisation of relations, that is to say, the process in which something newly formed - highly suggestive and attractive to the mind, and yet very ephemeral - can be strongly suggested to the mind in a more intelligible and clearer way, even if the newly formed idea is still very abstract or vague. Stated otherwise, abduction is the process through which a new perceived idea is given a 'local habitation and a name'. ${ }^{7}$ The conduct of a perceiving individual whose mind has perceived this emerging new idea may change in the event that this new element reveals itself as powerful enough to be further developed. This further development, which modifies conduct, is related to a modification of habit of feeling, action and thought. Each abductive inference produces, or at least has the potential to produce, a change of habit of conduct. However, the further pursuit of an idea formed by abduction implies some sort of testing and proving, even if the context is not a purely scientific one. In the context of pragmatism, pursuing the development of a newly formed idea or concept is related with an experiment. Every experiment must propel the hypostatisation proposed by abduction to the mind in some other form, embodied in some other medium. Generally stated, by developing a

6 Similarly as Peirce at the turning of the nineteenth to the twentieth century, Saussure made use of the concept of sign, a word derived from the

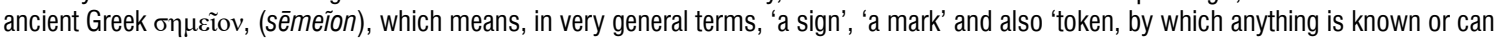
thereby be distinguished'. From this general definition, both thinkers sought to precise the specific meaning of sign according to their respective frameworks. As already pointed out, Saussure's notion of sign is that of a composite, part material, part ideally construed psychological entity, whereas for Peirce, sign is everything that acts as a medium, a third, a relational connector within a triadic, indecomposable and mediative relation.

7 The passage 'a local habitation and a name' is a reference to Shakespeare's A Midsummer Night's Dream. In relation to the Shakespearean quote, the new idea is presented with a more definite form and developed into a concept through its replication into some medium and through the articulation of a given language. 
new idea or a new concept and making it grow through successive tests, experiments and further embodiments regardless of the particular context of action - one engages in a pragmatic action.

This is what Peirce denominates as the third grade of clearness, which consists of the given representation that fruitful reasoning can be used in order to solve difficult conceptual and practical problems [16 p. 162]. Because of this, the third grade of clearness has a future-oriented character. Indeed, Peirce affirms that this grade of clearness, which he also calls pragmatistic adequacy and which is unveiled by methodeutic, imparts what 'ought to be the substance, or meaning, of the concept or other symbol in quotation, in order that its true usefulness may be fulfilled' (MS 649 1909, p. 2). ${ }^{8}$ It should be noted that the grades of clearness involved in the three branches of semiotics are not stages, 'as if one were done with before the next began' (MS 649 1909, p. 3). Peirce prefers to call them kinds because they are always present, running parallel to each other, forming thus bundles of semiotic operations. This is a characteristic feature of the precept of principle and data dependency in Peirce's architectonic philosophy: new principles and new data can always enter semiosis and the results obtained in the first study can be modified or enriched by the second, because details not included or not disclosed in the previous observations will be available in subsequent ones.

In an article entitled 'Issues of Pragmaticism' published in The Monist in 1905 [19], Peirce revisits his formulations of the maxim of pragmatism ${ }^{9}$ and reinforces the relationships expressed therein. At this point, however, he focuses on the clarification of the maxim by specifying the 'general modes of rational conduct' and the 'possible different circumstances'. Using these distinct expressions, Peirce complements earlier formulations of the maxim by making explicit the intellectual purport of a symbol, which has the power to generate a logical interpretant. Logical interpretants, according to Peirce's semiotics, have the character of a plan not only to furnish parameters for experimentation but also to produce new habits of conduct. This aspect of 'would be' of a logical interpretant becomes an integrative factor in pragmatism, for it can articulate these two modalities, that is, the general modes and the possible different conceivable circumstances that would arise under specific experiential conditions. Special attention has been given to the role of logical interpretants in developing arguments to develop a plan of conduct for future inquiries. The whole meaning of a concept, Peirce contends, is the general mental habit that consists of the production of these effects, that is, the effects that might conceivably have practical bearings. These effects are the possible contexts of possible different circumstances, which Peirce designates as modifying habits or implying capacities, and they are related to the whole meaning of the conception of the object. The whole meaning is the interpretational conception of these effects.

The formalisation of the maxim is still multifaceted, for it entails many of the important theoretical aspects encompassed by all three branches of semiotics, i.e., theory of inquiry, abduction, logical interpretant, intellectual concept and legisign. Fernando Zalamea, in his article 'A Category-Theoretic Reading of Peirce's System', contends that there are three complex webs through which the maxim of pragmatism filters positive experience: a modal web, a representational web and a relational web. Through these three webs, pragmatism is able to 'differentiate the one in the many' and, more importantly, to 'integrate the many in the one' [20 p. 205]. A given sign, which can potentially generate a logical interpretant, runs through the various contexts of representation able to interpret the sign. These various contexts of representation are related to the context of the possible. Within each context, it is necessary to disclose the practical consequents related to each representation. By examining these two studies in the contingencies of possible contexts and necessary contexts, potential relations between them become evident and relevant. Peirce's maxim of pragmatism, like his semiotics, reveals that knowledge is eminently contextual, not absolute; relational, not substantial; modal, not determinate; and synthetic, not analytic [20].

These aspects of transference, linkage and correlationality that characterise Peirce's maxim of pragmatism can be rendered more precise through the theoretical background of category theory. Moreover, this theoretical

8 The Charles S. Peirce papers, microfilm edition (Harvard University Library, Photographic Service, 1966). References use the numbering system for manuscripts (MS\#) developed by Robin in Ref. [17], as supplemented by Robin in Ref. [18]. For example, MS 649.2, 1910, indicates Robin's catalogue manuscript number 659 , page 2 , followed by the year of the writing.

9 The subject's broad scope makes it impossible for me, in this article, to cover the whole extent of the development of Peirce's maxim of pragmatism expressed in different forms and approaching different angles throughout his career. I have shown it elsewhere in connection with the study of design process read through Peirce's semiotics, although this study is far from complete [3]. Peirce proposed a myriad of formulations of his maxim of pragmatism, each time trying to cover some new aspect connected with a clearer understanding of it. In a formulation of 1907 , Peirce revisits his first formulation and rephrases it in light of his latest philosophical developments. He writes: 'Consider what effects that might conceivably have practical bearings, - especially in modifying habits or as implying capacities, - you conceive the object of your conception to have. Then your (interpretational) conception of these effects is the whole (meaning of) your conception of the object' (MS 3221907 pp. 11-12). 
background can also clarify the aspect of pragmatism as understood as a differential and integral abstract calculus.

A given sign, say, a highly suggestive set of poetic articulation, enters the process of semiosis. This sign will be represented in several forms - which can also be called subdeterminations - in possible contexts of interpretations. The necessary action-reactions involved in such interpretations enable the partial disclosure of some aspects of the sign, for this disclosure involves the deduction of practical consequences once a possibility of interpretation is rendered clearer. Furthermore, these aspects of the sign, while being interpreted in a myriad of possible contexts, contribute to what Zalamea calls the process of differentiation [20 p. 206].

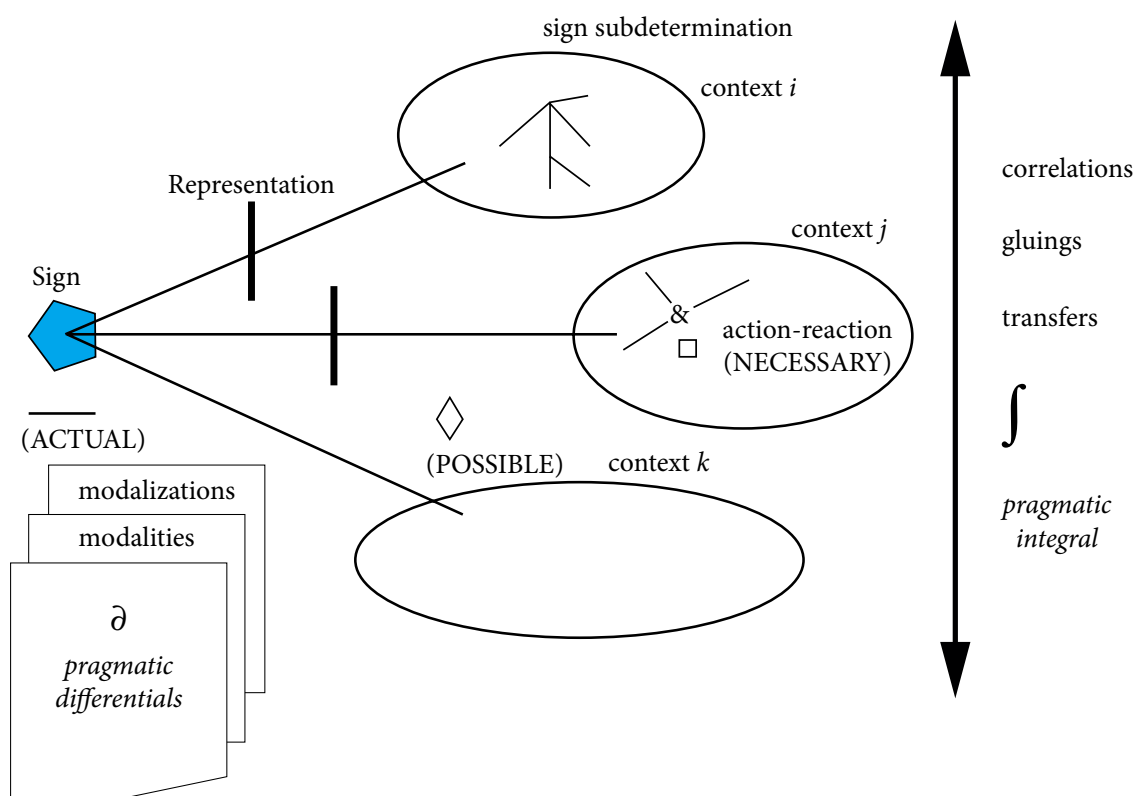

Fig. 2. The diagram exhibits a model of the fully modalised operation of pragmatism according to category theory.

The diagram (Fig. 2) expresses this process with the terms 'pragmatic differentials' and 'modalizations'. As Zalamea explains, the process indicates the possible variations of interpretation of a main leitmotif, that is, how the same motif 'can be extensively changed throughout the development of a musical composition' [20] and yet remain identifiable by its basic characteristics. Within this process of differentiation, the representation of a sign into another, more developed sign, implies subcategories such as fidelity, distance, reflexivity and partiality: this characterises the process of differentiation. This peculiar aspect of semiosis implies the interpretation of an intellectual concept, while this process is dispersed through multiple languages and multiple general modes of interpretation, as well as through a myriad of guidelines as to how information about these interpretants ought to be organised and stratified.

However, the most important aspect of Peirce's pragmatism is its capacity to propose the integration or reintegration of a new isolated or discovered element. After the sign in question has entered the process of semiosis and after it has been broken down into fragments in the myriad of possible interpretational contexts, these fragments become free to be correlated with each other and with other subfragments from previous logical analyses. The integrative character of methodeutic enables these correlations to take place; this in turn enables systemic linkages between fragments to arise, which then unveil potential new forms of knowledge. These new forms of knowledge were hidden in the first steps of the first representational process and could only have been enabled through connections of possible correlations by means of the discovery of analogies and transferences of structural layers. These become perceivable after the differentiation process has taken place [20]. The diagram (Fig. 2) exhibits this unique characteristic feature of Peircean pragmatism by identifying it as a pragmatic integral, which enables these correlations, gluing of fragments and transfers to become semiotically relevant.

In short, pragmatism's maxim is able to disclose the importance of local interpretation. However, its most important characteristic feature is the reconstruction of local interpretations through the operations of gluing and transferring differentiated representations, and integrating the differentiated fragments within new semiotic systems [20 p. 221]. 
Taking this and especially the type of future orientation made explicit by the maxim of pragmatism into account, it is possible to perceive why Peirce emphasised the idea of experiment, discovery and invention, and the formation of habits that would bring feeling, thought and conduct into relationship with one another. For Peirce, all branches of semiotics are traversed by abduction, from the evaluation of signs until the formulation of an experiment to clarify a difficult intellectual concept. In this sense, Peirce affirms that pragmatism is the very logic of abduction [21 pp. 226-241]. It would then follow that pragmatism, seen strictly as a logical maxim, should be tasked with the formulation of a sound hypothesis that indeed explains phenomena. A newly formulated and accepted hypothesis must, however, pass through the logical investigation as proposed by methodeutic so that the chosen hypothesis can be accepted as evaluated and proven. Peirce emphasises the thesis that pragmatism is the logic of abduction, by showing the role that hypotheses play within a scientific inquiry. He also points out the goodness of a hypothesis. His argument runs as follows: The goodness of anything is 'whether that thing fulfills its end' [21 p. 235]. Any given accepted hypothesis must, according to its end, be subjected to experiment. For Peirce, then, 'any hypothesis, therefore, may be admissible [...] provided it be capable of experimental verification, and only insofar as it is capable of such verification' [21 p. 235]. With this assertion, Peirce stresses the two main functions pragmatism should perform. ${ }^{10}$ First, pragmatism should rapidly dismiss all unclear ideas. Second, pragmatism's maxim should support and render distinct all ideas that are clear but difficult to apprehend. Both of these functions expected from pragmatism should, according to Peirce, 'take a satisfactory attitude toward the element of Thirdness' [21 p. 239], that is to say, in this case, to approach possible, and conceivable, interpretations of such poetic experience. This operation, here, is equivalent to the main articulation of pragmatism in relation to poeticity, which consists of the continual generation of new semiotical contexts, semioses and representations within the global context of poetic experience. Therefore, the character of poeticity is considered in a formative light, in which no system of language is previously assumed or given - but in formation, as a possibility of emerging from the methodeutical and pragmatic processes of differentiating and integrating.

Whenever a poetic experience enters the main stream of semiosis, it will act as a semantic trigger that, because of the aforementioned semantic tension created by the interaction of different elements associated together within a given context - such as a poem, picture, film, song and sculpture - will necessarily cause a pragmatic articulation. The experience of some poetic occurrence or event that happens to trigger this specific semiosis will be pervaded by a myriad of abductive relations, which are suggestions of significations and re-significations. These newly formed sign articulations are ever changing, oscillating, never definitive and evanescent but highly suggestive. As such, they have the nature of abductions, of possibilities of signification. They may be embodied into some different medium, articulated with a selected language - as in the example of the artist who is working with paint and canvas. Alternatively, the suggestions may be produced only at the level of emotional interpretants - the first level of interpretations of signs of evanescent possibilities that could be articulated into whatever future context they might be integrated. By context I mean, here, any possible form of expression not previously given but selected as the process of interpretation goes. For instance, a poetic experience of any kind may trigger special associations, qualitatively connecting two distinct sign systems, such as a symphonic composition and a painting process, with impressionist techniques. For the perceiver, this association can also be a rather poetic experience. A completely mundane experience may trigger traces of poetic qualities that, once they infuse the perceiver, set off a myriad of impulses to recreate new contexts, some mentally and some even physically by constructing or creating something, which, upon completion, will serve as a medium for further poetic experiences of a myriad of other observers. In light of Peirce's semiotics and pragmatism, poeticity can be considered as a poiesis in the making, in which sign processes and sign systems are constantly being created, re-contextualised, reformulated and poetically experimented upon.

\section{Conclusion}

With this analysis, I have used Peirce's semiotics and pragmatism to show that poetic experiences have the potential, as Roman Jakobson perceived and announced in his text 'Linguistics and Poetics', to extrapolate the dyadic boundaries of most structuralist studies of language. Poeticity will eventually emanate from its point of origin

10 Peirce states that if his theory of pragmatism is proven to be either wrong or incomplete, '(...) whatever the true doctrine of the Logic of Abduction may be ought to do these two services' (EP 2 p. 239, emphasis added). 
and pervade other linguistic and extra-linguistic processes of communication of any kind - codified and formative processes alike - and will constantly evade structural and rational description. I reiterate that no innumerous amount of rational explanations of a given poetic experience will ever exhaust the possibilities of that occurrence to generate new, previously unknown, meanings. Poetic occurrences will, at any rate, evade any attempts to drastically set the boundaries of the poetic contents in explanatory, exhaustive manners.

The key to a better grasp of poetic occurrences, experiences and phenomena is to consider them in their evanescent potential as well as prolific nature as the 'would be' of a certain kind, always possible of entering and pervading sign processes, but never definable enough to be fully denoted. It is through consideration of the pragmatic meaning of poetic experiences that the possible semiotical functioning of such poetic occurrence can be conceived. The connectivity thus rendered possible by the logic of abduction as the all-pervading operation of Peirce's pragmatism allows the mind to hypothetically synthesise relations between elements, which were never considered to have been in a relation to one another, thus grasping the possible - and in their very essence poetical - practical bearings of the boundless and free poetical meanings. Poeticity imposes itself upon sign operations and forces it to extrapolate the boundaries of codified language and strong-made, conventional correlations in order to enable an open search for qualities and similarities, contrapositions, new compositions, new associations and contradictions that will, upon this roaming free of poiesis, allow the settling back to a form of language that brings the poetics constructed into play. Consequently, sign processes formed as the outcome of poetic experiences are able to breach codified systems and overcome hardened semantic structures, thus poetically overcoming any form of hardened boundaries.

\section{Acknowledgements}

This paper was made possible by the Cluster of Excellence Image Knowledge Gestaltung. An Interdisciplinary Laboratory at the Humboldt Universität zu Berlin (sponsor number EXC 1027/1) with financial support from the German Research Foundation (Deutsche Forschungsgemeinschaft - DFG).

\section{References}

[1] Levin, R., 2010. Der Beitrag des Prager Strukturalismus zur Translationswissenschaft. Linguistik und Semiotik der literarischen Übersetzung. Berlin, Frank \& Timme Verlag für wissenschaftliche Literatur.

[2] Saussure, F. de., 2013. Cours de linguistique générale. Zweisprachige Ausgabe französischdeutsch mit Einleitung, Anmerkung und Kommentar von Peter Wunderli (Ed.). Tübingen, Narr Francke Attempto Verlag $\mathrm{GmbH}$.

[3] Da Costa e Silva, T., 2018. The logic of design process. Invention and discovery in light of the semiotics of Charles S. Peirce. Bielefeld: Transcript Verlag.

[4] Short, T. L., 2007. Peirce's theory of signs. Cambridge, Cambridge University Press.

[5] Nöth, W., 2000. Handbook der Semiotik. Stuttgart/ Weimar: Metzler.

[6] Eco, U., 1987. The influence of Roman Jakobson on the development of semiotics. Classics of Semiotics. New York: Plenum Press, 109-128.

[7] Jakobson, R., 1960. Linguistics and poetics. In Sebeok, T. (Ed.), Style in language. Massachusetts/
New York/London: Technology Press of the Massachusetts Institute of Technology/John Wiley \& Sons, Inc., 350-377.

[8] Cassirer, E., 1962. An Essay on man. New Haven, $\mathrm{CN}$ : Yale University Press.

[9] Jakobson, R., Pomorska, K., 1988 Dialogues. Cambridge, MA: MIT Press.

[10] Waugh, Linda R., 1980. The poetic function in the theory of Roman Jakobson. Poetics Today, 2(1), 57-82.

[11] Da Costa e Silva, T., 2016. A Linguagem Poética do Cinema de Animação. Lisbon: Nova Edições Acadêmicas.

[12] Hausman, C., 1989. Metaphor and art. Interactionism and reference in the verbal and nonverbal arts. Cambridge: Cambridge University Press.

[13] Eco, U., 1968. La Struttura Assente. Milano: Bompiani.

[14] Ehrat, J., 2005. Cinema \& semiotic. Peirce and film aesthetics, narration, and representation. Toronto/ Buffalo/London: University of Toronto Press.

[15] Lotman, Y., 1990. Universe of the mind. A semiotic 
theory of culture. Bloomington and Indianapolis, IN: Indiana University Press.

[16] Peirce, C S., 1897. The logic of relatives. The Monist, 7(2), 161-217.

[17] Robin, R., 1967. Annotated catalogue of the papers of Charles S. Peirce. Amherst, MA: University of Massachusetts Press.

[18] Robin, R., 1971. The Peirce papers: A supplementary catalogue. The Transactions of the Charles S. Peirce Society, 7(1), 37-57.

[19] Peirce, C S., 1905. Issues of pragmaticism. The Monist, 15(4), 481-499.
[20] Zalamea, F., 2010. A categorial-theoretic reading of Peirce's system: Pragmaticism, continuity, and the existential graphs. In Moore, M. (Ed.), New essays on Peirce's mathematical philosophy. Chicago/La Salle, IL: Open Court, pp. 203-234.

[21] Peirce, C S., 1998. The Essential Peirce: Selected Philosophical Writings, vol. 2. Houser, N., Kloesel, CJW. (Eds.) Bloomington: Indiana University Press, [References are abbreviated as "EP" followed by volume and page numbers]. 